\section{Propiedades y aplicación clínica de los ionómeros de vidrio de alta densidad disponibles en Lima-Perú}

\section{Properties and clinical application of high density glass ionomers available in Lima-Peru}

\section{Resumen}

El objetivo de la revisión fue conocer las propiedades y la aplicación clínica de los diferentes ionómeros de vidrio de alta densidad disponibles en Lima-Perú. Se realizó una revisión de la literatura científica desde enero 2010 hasta diciembre 2019 acerca de los ionómeros de vidrio de alta densidad. Esta revisión se realizó en las bases de datos SciELO, Pubmed y Google Scholar. Los descriptores para realizar la búsqueda fueron: cementos de ionómero de vidrio, ionómeros de vidrio de alta densidad y propiedades físicas, las combinaciones entre ellos y su equivalente en inglés. Se seleccionaron finalmente 40 artículos que cumplieron con los criterios de inclusión. Dentro de las marcas de ionómeros de alta densidad disponibles en Lima-Perú se encuentran: Ionofil® Plus (VOCO), KetacTM Molar Easymix (3M ESPE), Fuji IX GP (GC corporation) y EQUIA Forte ${ }^{\mathrm{TM}}(\mathbb{2}$ GC (Europe GC). Se presentan las propiedades y aplicación clínica de todos estos ionómeros. De acuerdo a la revisión de la literatura, de los cinco grupos estudiados: EQUIA Forte presentó una excelente adhesión al esmalte y la dentina. Fuji IX GP puede ser usado en restauraciones de Clase I y II, sin contactos oclusales y; EQUIA Forte puede utilizarse en pequeñas Clase II con /sin contactos oclusales, y con cuidado en grandes cavidades MOD siguiendo las recomendaciones del fabricante.

Palabras clave: Cementos de ionómero de vidrio; Cemento de polialquenolato de vidrio; Cementos dentales (fuente: DeCS BIREME).

\begin{abstract}
The objective of the review was to understand the properties and clinical application of the different high-density glass ionomers available in Lima, Perú. A review of the scientific literature about high-density glass ionomers, was conducted from January 2010 to December 2019. This review was performed in SciELO, Pubmed and Google Scholar databases. The descriptors used in the search were: glass ionomer cements, high density glass ionomers and physical properties, the combinations between them and their equivalent in English. Ultimately forty articles that met the inclusion criteria, were selected. The high-density ionomers available in Lima-Peru are Ionofil@ Plus (VOCO), KetacTM Molar Easymix (3M ESPE), Fuji IX GP (GC corporation) and EQUIA Forte ${ }^{\mathrm{TM}} \circledast$ GC (Europe GC). The properties and clinical application of all these ionomers are presented. According to the literature review, of the five groups studied; EQUIA Forte presented excellent adhesion to enamel and dentin. Fuji IX GP can be placed in Class II restorations
\end{abstract}

\section{Artículo de Revisión}

Rosalyn Mariela Caso Guerra ${ }^{1, a}$, Katherine Jeanette Campos Campos ${ }^{1, b}$

${ }^{1}$ Universidad Científica del Sur, Carrera de Estomatología, Lima, Perú.

a Cirujano Dentista.

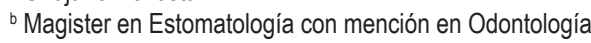
Pediátrica.

\section{Correspondencia:}

Rosalyn Mariela Caso Guerra: romaborletti@yahoo.es Av. Tupac Amaru Mz. 5 Lt.14, El Tambo- Huancayo ORCID: 0000-0002-1233-8565

\section{Coautor:}

Katherine Jeanette Campos Campos: kcampos@ cientifica.edu.pe ORCID: 0000-0002-9755-1743

\section{Editor:}

Antonieta Pérez-Flores

Universidad de Concepción, Chile

Conflicto de intereses: no existe conflicto de interés.

Financiamiento: autofinanciado.

Recibido: $13 / 03 / 21$

Aceptado: 26/07/21

Publicado: 05/10/21 
without occlusal contacts and EQUIA Forte can be used in small Class II with / without occlusal contacts, and cautiously in large MOD cavities following the manufacturer's recommendations.

Keywords: Glass ionomer cement; Glass Polyalkenoate Cement; Dental cements (source: MeSH NLM).

\section{Introducción}

La Odontología restauradora moderna está orientada a la extracción mínima de tejido dental y el uso de materiales con posible acción terapéutica sobre la dentina desmineralizada. Requisitos que encajan perfectamente con el ionómero de vidrio ${ }^{1}$. El término" ionómero de vidrio" es el más usado en odontología, pero no el correcto, según los compiladores de Chemical Abstracts el término "cemento de polialquenato de vidrio" es el más idóneo ${ }^{2,3}$.

El ionómero de vidrio fue inventado por Alan Wilson ${ }^{2,3}$ y Brian Kent en 1969 y desarrollado por McLean y Wilson ${ }^{3,4}$ a principios de los 70, como resultado de estudios para mejorar el cemento de silicato ${ }^{5}$. En 1972, se realizó la primera publicación en el British Dental Journal. En Europa (1975), sale el primer ionómero de vidrio con el nombre de ASPA ${ }^{6}$ (aluminio, silicato, poliacrílico), a principios de 1977, llegó a Estados Unidos y hacia finales de la década de 1970, a latinoamérica ${ }^{6}$. Desde entonces hasta el presente, este material ha evolucionado ${ }^{6,7}$.

El ionómero de vidrio (IV) se puede definir como un material a base de agua, resultante de una reacción ácido-base ${ }^{8}$, entre un ácido polialquenoico y un vidrio de aluminosilicato básico (ASG) ${ }^{9,10}$, que genera un polisal y forma la matriz inicial del cemento ${ }^{11}$. El ácido polialquenoico suele ser un ácido poliacrílico, ácido (politacónico, polimaleico) o un copolímero de los anteriores. El ASG contiene $\mathrm{Ca} 2$ +, $\mathrm{Al} 3$ + y Si 4 + como componentes esenciales, unidos entre sí mediante puentes de oxígeno. Otros iones, como F- y PO4 3- se suelen agregar a la composición de vidrio ${ }^{11}$. La versatilidad del ionómero de vidrio lo convierte en un material restaurador importante por sus propiedades únicas como: excelente biocompatibilidad, coeficiente de expansión térmica cercano al de los dientes ${ }^{12-14}$ adhesividad química a los tejidos dentales, la capacidad de remineralizar los tejidos dentales mediante la liberación de flúor a largo plazo 9,15-17 (efecto anticariogénico), estabilidad dimensional, insolubilidad en fluidos orales a temperaturas intraorales lo que lo hace muy apropiado para su uso en niños ${ }^{18}$. La absorción de iones fosfato y calcio de la saliva le proporciona dureza al material ${ }^{19}$. Tienen la capacidad constante de adquirir y liberar fluoruros de diferentes fuentes del medio bucal ${ }^{20}$.

Uno de los principales inconvenientes de los ionómeros de vidrio son sus propiedades mecánicas débiles como: la tenacidad, la fragilidad y la baja resistencia a la compresión, por lo que se han investigado materiales de relleno alternativo ${ }^{9}$. A principios de la década de 1990, se desarrollaron los cementos de IV de alta viscosidad o densidad, como materiales de obturación en la técnica de terapia restaurativa atraumática (TRA); deseables debido a sus propiedades únicas de fraguado más rápido, resistencia adecuada y pulible en una sola cita, aunque las resinas siguen siendo superiores en cuanto a resistencia a la fractura ${ }^{21}$. Se puede definir a los ionómeros de alta densidad como ionómeros convencionales cuyos vidrios han sido mejorados ${ }^{6}$; donde el Calcio (Ca) ha sido reemplazado por Estroncio (Sr) e incluso, Zirconio $(\mathrm{Zr})$ ${ }^{21}$, logrando propiedades físicas superiores al optimizar la distribución del tamaño de sus partículas y poliácidos, lo que resulta en una matriz con alta reticulación ${ }^{1}$.

Las modificaciones de los ionómeros convencionales para mejorar sus propiedades mecánicas y formar ionómeros de alta densidad han incluido el uso de poliácidos alternativos, poliácidos deshidratados activados por agua, cerámicos, adiciones de metal, partículas de vidrio de tamaño más pequeñas, agentes antibacterianos y nuevas composiciones de vidrio ${ }^{5}$. Además, del tipo, la cantidad y la masa molecular con alta concentración de PAA (ácido poliacrílico) ${ }^{19}$ y la relación polvo / líquido $(\mathrm{P} / \mathrm{L})^{20,22,23}$. Se considera que todos los ionómeros con una relación $\mathrm{P} / \mathrm{L}$ igual o inferior a 2:1, son considerados ionómeros de baja viscosidad ${ }^{15}$.

Dada la gran variedad de IV disponibles en Lima-Perú, es importante realizar esta revisión, pues, permitirá actualizar al lector sobre los IV de alta densidad disponibles en el mercado, describir sus propiedades e indicaciones. Lo que permitirá al odontólogo, elegir el material más adecuado para su paciente, tratando de aprovechar las ventajas del material, disminuyendo el tiempo de trabajo y logrando confort en el paciente.

Por ello, el propósito de esta revisión fue describir las propiedades y la aplicación clínica de los diferentes ionómeros de vidrio de alta densidad disponibles en Lima- Perú.

\section{Metodología}

Métodos de búsqueda. Se realizó una revisión de la literatura científica desde enero 2010 hasta diciembre 2019. Esta revisión se realizó en las bases de datos Scielo, Pubmed y Google Scholar. Los descriptores para realizar la búsqueda fueron: cementos de ionómero de vidrio, ionómeros de vidrio de alta densidad y propiedades físicas, las combinaciones entre ellos y su equivalente en inglés. Los criterios de inclusión para la selección de la bibliografía fue que los artículos mencionaran alguna de las marcas de IV de alta densidad que se comercializan en Lima- Perú. Se descartaron las revisiones de literatura, revisiones sistemáticas y meta-análisis; se descartó también la literatura gris, por lo que fueron finalmente incluidos 40 artículos. 
Selección de ionómeros de vidrio disponibles en $\mathrm{Li}$ ma-Perú. Se realizó una visita a las principales tiendas de productos dentales ubicadas en la ciudad de Lima preguntando ¿Qué ionómero de vidrio de alta densidad para restauración vendían? y así, conocer las marcas comerciales disponibles en nuestro medio. De todas ellas, se seleccionaron solo las marcas que cumplían con los requisitos de ionómeros de vidrio de alta densidad según los artículos encontrados. Quedando seleccionadas las siguientes marcas: Ionofile Plus (VOCO), KetacTM Molar Easymix (3M ESPE), Gc Fuji IX GP (GC corporation) $y$ Equia Forte ${ }^{\circledR}$ GC (Europe GC).

\section{Resultados}

Dentro de las marcas de ionómeros de alta densidad disponibles en Lima- Perú se encuentran: Ionofil® Plus (VOCO), KetacTM Molar Easymix (3M ESPE), Fuji IX GP (GC corporation) y EQUIA Forte ${ }^{\mathrm{Tm}} \circledast \mathrm{GC}$ (Europe GC). La composición, indicaciones y propiedades de estos IV de alta densidad se muestran en las tablas 1 y 2.

\section{Discusión}

Los IV de alta densidad son utilizados en la técnica restaurativa atraumática (TRA) de eliminación manual de caries $^{2,23}$, la Organización Mundial de la Salud (OMS) en 1994 reconoce el TRA como un nuevo enfoque para el tratamiento dental en regiones económicamente menos favorecidas ${ }^{21}$. También, se pueden emplear los ionómeros como materiales restauradores de larga duración especialmente, en la dentición primaria, como revestimientos y bases, como selladores de presión y como agentes de unión para brackets de ortodoncia ${ }^{18}$. Por su adhesión química a las estructuras dentales, su biocompatibilidad, su liberación fluoruros por largos períodos de tiempo ${ }^{24}$ y por su coeficiente de expansión térmica cercano al de los dientes, son ideales en niños ${ }^{25}$. En estudios realizados, se ha observado una tasa de supervivencia el $82 \%$ con la técnica TRA y $24 \%$ en oclusoproximal durante 3 años ${ }^{22}$.

El ionómero de vidrio es un material que actualmente se utiliza en tratamientos restauradores en molares temporales y molares permanentes jóvenes ${ }^{2}$. Los procesos de maduración del polialquenoato se completan esencialmente a las 24 horas ${ }^{5}$. Presenta baja erosión ácida, liberación alta de fluoruros durante las primeras 24 horas y disminuye durante los siguientes 15 días a medida que se forma la matriz ${ }^{12,15,24}$. Existe interacción química entre los IV de alta densidad y la dentina, cuanto mayor sea la cantidad de dentina, mayor será la banda asociada al fosfato, ya que este mineral es abundante en la dentina inorgánica ${ }^{8}$. La densidad de túbulos más baja en la dentina superficial proximal y oclusal da como resultado un mejor rendimiento de la fuerza de unión a los IV de alta densidad (Fuji IX o Ketac Molar) ${ }^{22}$.

Los materiales con la mayor proporción de polvo a líquido tienen las mejores propiedades mecánicas ${ }^{18,21}$. La relación polvo/líquido influye en la liberación de fluoruro como en la erosión ${ }^{15}$. La exposición de la crema dental acidulada no estimula la liberación de flúor de los IV ${ }^{14}$.

Al comparar los cinco cementos de IV convencionales para restauración de diferentes marcas, se determinó en la prueba de tiempo de fraguado, que todas las marcas cumplieron con los valores establecidos en la Norma No 96 de la ADA. En la prueba de resistencia a la compresión, sólo Fuji II $\circledast$ y Ketac Molar Easymix ${ }^{\circledR}$ alcanzaron valores superiores a los $130 \mathrm{Mpa}$; tres de los cinco cementos estudiados no alcanzaron el valor mínimo de resistencia a la compresión establecido en la Norma $\mathrm{N}^{\circ} 96$ de la ADA, prueba fundamental ionómeros de vidrio restauradores ${ }^{26}$.

Se encontró que el Ketac TM Molar Easy Mix e Ionofil Molar ${ }^{\circledR}$ mostraron menores valores de microfiltración con la jeringa centrix siendo el Ketac TM Molar Easy Mix el que obtuvo menores valores de microfiltración ${ }^{27}$.

El ionómero Fuji IX presentó mejor resistencia a la compresión y resistencia a la tracción que Ketac Molar ${ }^{28}$. El Fuji IX tiene un valor de resistencia a la compresión vertical casi dos veces mayor que el Ketac Molar ${ }^{29}$ los valores de resistencia a la compresión vertical proporcionados por este estudio sugieren la posibilidad de utilizar

Tabla 1. Composición de los ionómeros de vidrio de alta densidad disponibles en Lima-Perú

\begin{tabular}{|c|c|c|}
\hline Material & Composición & Polvo / líquido \\
\hline $\begin{array}{l}\text { Ionofil@ Plus } \\
\text { (VOCO) }\end{array}$ & $\begin{array}{l}\text { Polvo: Vidrio micronizado (vidrio de fluorosilicato, } \mathrm{Sr}, \mathrm{Al}, \mathrm{Ca} \text { ) y pigmen- } \\
\text { to (dióxido de titanio y óxido de hierro) }{ }^{15} \text {. } \\
\text { Líquido: ácidos policarboxílicos y tartáricos y agua desionizada }{ }^{15} \text {. }\end{array}$ & $4,7-5,6: 1$ \\
\hline $\begin{array}{l}\text { Ketac Molar Easymix }{ }^{\circledR} \\
\text { (3M ESPE }\end{array}$ & $\begin{array}{l}\text { Polvo: Vidrio micronizado } \mathrm{Sr} \text {, (vidrio de fluorosilicato } \mathrm{Al}, \mathrm{Ca}, \mathrm{La} \text { ) y pig- } \\
\text { mento (dióxido de Ti y óxido de aluminio }{ }^{15,21,32,34} \text {. } \\
\text { Líquido: } 5 \% \text { copolímero de ácido acrílico, ácido maléico, agua y ácido } \\
\text { tartárico. }\end{array}$ & $4,5: 1$ \\
\hline $\begin{array}{l}\text { Fuji IX GP®) } \\
\text { (GC Europe) }\end{array}$ & $\begin{array}{l}\text { Polvo: Vidrio micronizado de (alumino-fluoro-silicato). Líquido: ácido } \\
\text { poliacrílico, agua destilada, ácido carboxílico polibásico. }{ }^{15,21,32,34}\end{array}$ & $3,6: 1$ \\
\hline $\begin{array}{l}\text { EQUIA Forte }{ }^{\mathrm{TM} @} \\
\text { (GC Europe) }\end{array}$ & $\begin{array}{l}\text { Equia Fil: Polvo: } 95 \% \text { de vidrio de fluoroalumino-silicato de estroncio, } 5 \% \\
\text { de ácido poliacrílico Líquido: } 40 \% \text { de ácido poliacrílico acuoso } 1,15,32,34,35 \\
\text { Equia Coat Resina de recubrimiento superficial de baja viscosidad nanofi- } \\
\text { llizada } 50 \% \text { de metacrilato de metilo, 0,09\% de alcanforquinona. }\end{array}$ & \\
\hline
\end{tabular}


Tabla 2. Indicaciones y propiedades de los ionómeros de vidrio de alta densidad disponibles en Lima- Perú

\begin{tabular}{|c|c|c|}
\hline Material & Indicaciones & Propiedades \\
\hline $\begin{array}{l}\text { Ionofil@ Plus } \\
\text { (VOCO) }\end{array}$ & $\begin{array}{ll}\text { - } & \text { Técnica “TRA" } \\
\text { - } & \text { Dientes primarios en: Clase I, V. } \\
& \text { Dientes permanentes: Clase I (peque- } \\
\text { nas), Clase III y en fosas y fisuras } \\
\text { - Disponible en tres tonos: A1, A2 y A3 }{ }^{36} .\end{array}$ & $\begin{array}{l}\text { - } \quad \text { Consistencia esparcible. } \\
\text { - } \quad \text { Buena adaptación. } \\
\text { - } \quad \text { Buy buen fluido } \\
\text { - } \quad \text { Buena estética }{ }^{27} \text {. } \\
\text { - } \quad \text { Presenta menoción al esmalte y dentina }{ }^{6} \text {. } \\
\text { rante las primeras } 24 \text { horas y disminuye durante los siguientes } \\
15 \text { días }{ }^{12} \text {. }\end{array}$ \\
\hline $\begin{array}{l}\text { Ketac Molar Easymix® } \\
\text { (3M ESPE) }\end{array}$ & $\begin{array}{l}\text { - Técnica "TRA", } \\
\text { - } \quad \text { Dientes primarios en: Clase I y V }{ }^{37} \text {. } \\
\text { Dientes permanentes como provisio- } \\
\text { nal: Clase I(pequeñas), III y en fosas y } \\
\text { fisuras. } \\
\text { - Técnica sándwich con composite. } \\
\text { - Reconstrucción de muñones o piezas } \\
\text { con tratamiento endodóntico. Ideal } \\
\text { para Odontología Mínimamente Inva- } \\
\text { siva (MID). } \\
\text { Disponible en } 4 \text { tonos: (A1, A3, A4 y } \\
\text { B2) }\end{array}$ & $\begin{array}{l}\text { - } \quad \text { Fácil y rápido manejo }{ }^{28} \text {. } \\
\text { Es menos viscoso, facilitando la aplicación en fosas y fisuras } \\
\text { para los odontopediatras }{ }^{38} \text {. } \\
\text { Baja erosión ácida }{ }^{22,18} \text { y liberación de flúor prolongada que } \\
\text { disminuye durante los siguientes } 15 \text { días }^{12,25} \text {. } \\
\text { Radiopaco }{ }^{28} \text {. } \\
\text { - Integración al esmalte es regular, pero la integración a la den- } \\
\text { tina es mejoror. A mayor cantidad de dentina, mayor probabili- } \\
\text { dad de formar nuevas bandas }{ }^{8} \text {. } \\
\text { Alta resistencia a la flexión, alta resistencia a la compresión }{ }^{18,33} \text {, } \\
\text { cuyo valor es } 175 \mathrm{Mpa}^{18} \text {. La resistencia a la compresión vertical } \\
\text { es de } 41 \mathrm{Mpa}^{31} \text {. }\end{array}$ \\
\hline $\begin{array}{l}\text { Fuji IX GP®) } \\
\text { (GC Europe) }\end{array}$ & $\begin{array}{ll}\text { - } & \text { Técnica "TRA" } \\
\text { - } & \text { Dientes primarios: clase I y II. } \\
& \text { Dientes permanentes de clase I y II } \\
& \text { sin cargas }{ }^{7,11,13} \text {. Clase V y superficies } \\
& \text { radiculares; } \\
\text { - } & \text { Reconstrucción de muñones, técnica } \\
& \text { sándwich con composite }{ }^{30} \text {. } \\
\text { - } \quad \text { En fosas y fisuras }{ }^{38} \text {. } \\
\text { Disponible en } 3 \text { tonos estéticos (A2, } \\
\text { A3, A3.5 })^{38} \text {. }\end{array}$ & $\begin{array}{l}\text { - } \quad \text { Fácil de usar y colocación en un solo paso }{ }^{30} \text {. } \\
\text { - } \quad \text { Buena radiopacidad }{ }^{30} \text {. } \\
\text { - } \quad \text { Liberente biocompatibilidad y buen sellado marginal }{ }^{17} \text {. } \\
\text { disminuye los siguientes } 15 \text { días }^{12} \text {. } \\
\text { - } \quad \text { Mejor resistencia a la compresión y tracción que Ketac Molar } \\
\quad \text { - } \quad \text { Resistencia a la compresión vertical es de } 76 \mathrm{Mpa}^{31} \text {. }\end{array}$ \\
\hline $\begin{array}{l}\text { EQUIA Forte }{ }^{\mathrm{TM}} \mathbb{R} \\
\text { (GC Europe) }\end{array}$ & $\begin{array}{l}\text { - } \quad \text { Técnica “TRA". } \\
\text { Restauración para todas las edades: } \\
\text { Restauraciones de Clase I, II }{ }^{13} \mathrm{e} \\
\text { incluye restauraciones Clase II que } \\
\text { soportan cargas oclusales }{ }^{7,11} \text { y Clase } \\
\text { V } 35 \text {. } \\
\text { - Útil en pacientes con bruxismo }{ }^{33} \text {. } \\
\text { - } \quad \text { Excelente para restaurar molares } \\
\text { permanentes en pacientes con MIH } \\
\text { - } \quad \text { Disponible en } 8 \text { tonos estéticos. } \\
\text { (A1,A2,A3,A3.5,B1,B2,B3 y C4) }{ }^{40} \text {. }\end{array}$ & $\begin{array}{l}\text { - } \quad \text { Fácil y rápido manejo, no pegajoso y empacable, sin } \\
\text { polimerización, buen sellado marginal }{ }^{32} \text {. } \\
\text { - } \quad \text { Es encapsulado de alta densidad }{ }^{35} \text {. } \\
\text { - } \\
\text { - } \quad \text { pero liberación de flúor durante las primeras } 24 \text { horas } \\
\text { - } \quad \text { Tiene excelonte resistencia al desgaste y a la erosión ácida }{ }^{32} \text {. } \\
\text { - } \quad \text { Mejor resistencia a la compresión, a la tracción, a la flexión } \\
{ }_{35,37} \text { y dureza superficial que Fuji IX }{ }^{10,23} \text {. El agente de recubri- } \\
\text { miento agregado contribuye al aumento de la resistencia a las } \\
\text { fuerzas mecánicas }{ }^{35} \text {. } \\
\text { Tiene valores altos de resistencia a la tracción diametral a la } \\
\text { compresión }{ }^{36} \text { y a la flexión }\end{array}$ \\
\hline
\end{tabular}

estos materiales en dientes temporales con caries de clase I y II Black en niños entre 8 y 10 años y para los dientes temporales que permanecen más tiempo en la arcada dental, debemos hacer otra elección de material ${ }^{29}$. Los sellantes de fosas y fisuras realizados con Fuji IX tuvieron una tasa de sobrevivencia del doble de tiempo con respecto al ionómero de bajo precio ${ }^{30}$. Hay mayor fracaso en clase II de tres superficies (MOD), que en clase II de dos superficies. La razón se puede atribuir a la baja resistencia a la flexión de los IV en general, que es un problema en las cavidades y no en los empastes de una sola superficie ${ }^{16}$.

El ionómero EQUIA Forte Fil es un material restaurador prometedor con resistencia a la flexión y dureza superficial superiores en comparación con su predecesor, el Fuji IX GP u otros ionómeros de vidrio disponibles ${ }^{12,30-32}$. En una evaluación sobre el rendimiento clínico, al cabo de 4 años, el EQUIA Fil GC y Fuji IX GP $\circledast$ fast, ambos funcionan bien en cavidades Clase I y Clase II, pero en cavidades clase II grandes habrá más probabilidad de falla del material ${ }^{1}$. Se presenta un mayor fracaso en cavidades clase II de tres superficies (MOD), que en clase II de dos superficies. La razón se puede atribuir a la baja resistencia a la flexión de los IV en general. El ionómero EQUIA Forte con un recubrimiento de resina nanofill mostró un mejor rendimiento ${ }^{1,16}$. Es excelente para restaurar las molares permanentes con MIH con la técnica TRA ${ }^{39}$, útil en restauraciones en pacientes con bruxismo ${ }^{33}$.

En un estudio de 2017, se valora a cuatro ionómeros de alta densidad. De los cuatro grupos estudiados, el EQUIA Fil y el EQUIA Forte fueron los que presentaron una excelente adaptación marginal. En el caso del Ketac Molar, la integración con el esmalte fue regular, pero la adaptación a la dentina fue muy buena. En el caso de Ionofil plus; tanto la adaptación al esmalte como a la dentina, fueron buenas ${ }^{6}$.

Los IV encapsulados tienen valores más altos de resistencia a la tracción diametral, a la flexión y a la compresión 
que los IV de alta viscosidad mezclados a mano comúnmente utilizados ${ }^{32}$.

\section{Conclusiones}

Se describieron las propiedades y aplicación clínica de cinco ionómeros de vidrio de alta densidad disponibles en Lima-Perú, encontrándose que el EQUIA Forte presenta mejor adaptación marginal, resistencia a flexión, compresión, dureza superficial y resistencia a la tracción que los otros ionómeros estudiados. El sistema EQUIA Forte y el ionómero Fuji IX GP funcionan bien en restauraciones Clase I y II sin contactos oclusales, pero EQUIA Forte se puede usar en Clase II pequeñas con contactos oclusales. También, podría ser usado en cavidades grandes MOD siguiendo las recomendaciones del fabricante. El Ketac Molar Easimix y el Ionofil Plus Voco pueden ser usados en cavidades clase I pequeñas de molares primarias.

\section{Referencias bibliográficas}

1. Gurgan S, Kutuk ZB, Ergin E, Oztas SS, Cakir FY. Four-year Randomized Clinical Trial to Evaluate the Clinical Performance of a Glass Ionomer Restorative System. Operative Dentistry. 2015;40-2:134-143. DOI: 10.2341/13-239-C.

2. Torres MG, Mendoza TR, Guerrero IJ, Yamamoto NJ. Evaluación de la resistencia a la erosión ácida de dos ionómeros de vidrio utilizados en la técnica restaurativa atraumática (TRA), modificados con un antibiótico. Rev Cient Odontol (Lima). 2015;3(2):326-332. DOI: https://doi. org/10.21142/2523-2754-0302-2015-326-332

3. McLean JW, Nicholson JW, Wilson AD. Proposed nomenclature for glass-ionomer dental cements and related materials. Quintessence. 1994;25(9):587-9.

4. Webman M, Mulki E, Roldan R, Arevalo O, Roberts J, Garcia-Godoy F. A retrospective study of the 3-year survival rate of resin-modified glass-ionomer cement class II restorations in primary molars. J Clin Pediatr Dent. 2016;40(1):8-13. DOI: 10.17796/1053-4628-40.1.8

5. Moberg M, Brewster J, Nicholson J, Roberts H. Physical property investigation of contemporary glass ionomer and resin-modified glass ionomer restorative materials. Clin Oral Investig. 2019;23(3):1295-308. DOI: $10.1007 / \mathrm{s} 00784-018-2554-3$

6. Cedillo VJJ, Herrera AA, Farías MR. Enamel and dentin hybridization of high density glass ionomers; SEM study. Rev ADM. 2017;74(4):177-184. Disponible en: https://www.medigraphic.com/cgi-bin/new/resumenI. cgi? IDARTICULO=74115

7. Chalissery VP, Marwah N, Almuhaiza A, AlZailai A, Chalisserry E, Bhandiet $S$ et al. Study of the Mechanical Properties of the Novel Zirconia- reinforced Glass Ionomer Cement. J Contemp Dent Pract. 2016;17(5):394-8. DOI: $10.5005 /$ jp-journals-10024-1861

8. Yamakami SA, Mori A, Sato F, Medina A, Correa R, Baesso M. Study of the chemical interaction between a high-viscosity glass ionomer cement and dentin. J Appl Oral Sci. 2018;26(44). DOI: 10.1590/1678-77572017-0384
9. De Souza BM, Moreli D, Magalhães A. Antimicrobial and anti-Caries effect of new glass ionomer cement on enamel under microcosm biofilm model. Braz Dent J. 2018;29(6):599-605. DOI: 10.1590/01036440201802163

10. Hernández R, Moraga R, Velásquez M, Gutiérrez F. Resistencia compresiva vidrio ionómero Iono fil Molar® y Vitremer® según tiempo de exposición en saliva artificial. Rev. Clin. Periodoncia Implantol. Rehabil. Oral Vol. 2013;6(2);75-77. DOI: 10.4067/ S0719-01072013000200005.

11. Deb S, Nicholson JW. The effect of strontium oxide in glass-ionomer cements. J Mater Sci Mater Med. 1999;10(8):471-4. DOI: $10.1016 / j . p r o s-$ dent.2019.03.012

12. Moshaverinia M, Navas A, Jahedmanesh N, Shah K, Moshaverinia A, Ansari S. Comparative evaluation of the physical properties of a reinforced glass ionomer dental restorative material. J Prosthet Dent. 2019;122(2):15459. DOI: $10.1016 /$ j.prosdent.2019.03.012

13. Zoergiebel J, Ilie N. Evaluation of a conventional glass ionomer cement with new zinc formulation: Effect of coating, aging and storage agents. Clin Oral Investig. 2013;17(2):619-26. DOI: 10.1007/s00784-012-07331

14. Gomes V, Vidal A, Simóes K, Souza M, Pereira P, Sampaio $\mathrm{F}$ et al. Evaluation of acidulated dentifrice influence on fluoride releasing from glass-ionomer cements. Brazilian Dent Sci. 2015;18(3):84. DOI: 10.14295/ bds.2015.v18i3.1134

15. Bueno L, Silva R, Magalhães A, Navarro M, Pascotto $\mathrm{R}$, Buzalaf $\mathrm{M}$ et al. Positive correlation between fluoride release and acid erosion of restorative glass-ionomer cements. Dent Mater. 2019;35(1):135-143. DOI: 10.1016/j.dental.2018.11.007

16. Klinke, T, Daboul A, Turek A, Frankerberger R, Hickel $\mathrm{R}$, Biffard R. Clinical performance during 48 months of two current glass ionomer restorative systems with coatings: a randomized clinical trial in the field. Trials. 2016;17:239. DOI: 10.1186/s13063-016-1339-8

17. Iftikhar N, Devashish, Srivastava B, Gupta N, Ghambir N, Rashi-Singh. Comparative Evaluation of Mechanical Properties of Four Different Restorative Materials: An In Vitro Study. Int J Clin Pediatr Dent. 2019;12(1):47-9. DOI: $10.5005 /$ jp-journals-10005-1592

18. Murthy SS, Murthy GS. Comparative Evaluation of Shear Bond Strength of Three Commercially Available Glass Ionomer Cements in Primary Teeth. J Int Oral Health 2015;7(8):103-7. Disponible en: https://www. ncbi.nlm.nih.gov/pmc/articles/PMC4588773/

19. Hasan A, Sidhu S, Nicholson J. Fluoride release and uptake in enhanced bioactivity glass ionomer cement ("glass carbomer ${ }^{\mathrm{TM}}$ ") compared with conventional and resin-modified glass ionomer cements. J Appl Oral Sci. 2019; 27:1-6. DOI: 10.1590/1678-7757-2018-0230

20. De Caluwé T, Vercruysse C, Ladik I, Convents R, Declercq H, Martens L, Verbeeck R. Addition of bioactive glass to glass ionomer cements: Effect on the physico-chemical properties and biocompatibility. 
Dent Mater. 2017;33(4):e186-e203. DOI: 10.1016/j. dental.2017.01.007

21. Abraham D, Thomas AM, Chopra S, Koshy S. A Comparative Evaluation of Microleakage of Glass Ionomer Cement and Chitosan-modified Glass Ionomer Cement: An in vitro Study- Int J Clin Pediatr Dent. 2014;7(1):610. DOI: $10.5005 /$ jp-journals-10005-1225

22. Tadesco T, Calvo A, Domingues G, Mendes F, Raggio D. Bond Strength of High-Viscosity Glass Ionomer Cements is Affected by Tubular Density and Location in Dentin? Microsc. Microanal. 2015;21(04):849-854. DOI: $10.1017 /$ S1431927615013021

23. Machado J, Duque C, Pitzer J, Scarparo A. Effect of storage time and chlorhexidine addition on the mechanical properties of glass ionomer cements. Brazilian J Oral. 2018;16:1-9. DOI: 10.20396/bjos.v16i0.8650457

24. Delgado C, Ramírez J,Yamamoto A. Liberación de fluoruro de dos cementos de ionómero de vidrio: estudio in vitro. Rev Odont Mex. 2014;18(2):84-8.

25. Lakshmi S, Sahana S, Kumar A, Prasad G,Bezawada S, Deepika M. Atraumatic restorative treatment vs. Hall technique for occlusoproximal lesions in primary dentition-an in vivo study. J Clin Diagnostic Res. 2018;12(2):ZC09-ZC13. DOI: 10.7860/ JCDR/2018/29576.11186.

26. Flores L, Ramírez J. Ionómeros de vidrio restauradores: valoración de acuerdo a la Norma 96 de la ADA. Revista ADM. 2010;67(2):72-77.

27. Basurto-Sampedrano KE, Barragán-Salazar NC. Comparación de la microfiltración del Ketac ${ }^{\mathrm{TM}}$ Molar e Iono fil Molar ${ }^{\circledR}$ con centrix y espátula TRA Comparison of the dental leakage between Ketac ${ }^{\mathrm{TM}}$ Molar e Iono fil Molar ${ }^{\circledR}$ with centrix and ART spatula. Ces Odontol. 2016;29(2):5-11. DOI: 10.21615/cesodon.29.2.1

28. Patel A, Dalal D, Lakade L, Shah P, Caudhary S, and Lodaya R. Comparitive evaluation of compressive strength and diametral tensile strength of zirconomer, ketac molar and type compressive strength and diametral tensile strength of zirconomer, ketac molar and type IX GIC - an in -vitro study. International Journal of $\mathrm{Cu}-$ rrent Research. 2018;10(06):70091-4. Disponible en: https://www.journalcra.com/article/comparitive-evaluation-compressive-strength-and-diametral-tensile-strength-zirconomer-ketac.

29. Lile I, Cornel P, Hosszu T, Vasca E, Vasca V, Bungau S et al. A subsidiary physical research of glass ionomers. Mater Plast. 2015;52(2):175-9.

30. Hesse D, Calil C, Brandão C, Da Franca C, Medeiros F, Raggio D. Low-cost glass ionomer cement as ART sealant in permanent molars: a randomized clinical trial. Braz Oral Res. 2015;29(1):1-9, DOI: 10.1590/18073107BOR-2015.vol29.0063
31. Cedillo Valencia JJ, Herrera Almanza A, Cedillo Valencia F. Caso clínico: Innovación del futuro en obturación de cavidades equia forte. The future in cavity obturation. Revista de operatoria dental y biomateriales. 2017;6(1):1-11. [Consultado el 17 de agosto 2021]. Disponible en: https:/www.studocu.com/co/document/ universidad-el-bosque/periodoncia/articulo-ionomero-de-vidrio/3789830

32. Molina G, Cabral R, Mazzola I, Lascano L, Frencken J. Mechanical performance of encapsulated restorative glass-ionomer cements for use with Atraumatic Restorative Treatment (ART). J Appl Oral Sci. 2013; 21 (3): 243-9. DOI: 10.1590/1679-775720130129.

33. Vural U, Meral E, Ergin E, Gürgan S. Twenty-four-month clinical performance of a glass hybrid restorative in non-carious cervical lesions of patients with bruxism: a split-mouth, randomized clinical trial. Clin Oral Invest. 2020;24:1229-1238. DOI:10.1007/s00784-01902986-x.

34. Bissoto A, Kicuti A, Kerber T, Minatel M, Prócida D. Evaluation of the relationship between the cost and properties of glass ionomer cements indicated for atraumatic restorative treatment. Braz Oral Res. 2016;30(1):3-9.

35. GC America | EQUIA Forte®-Liberación de flúor, restaurador de GH. [Consultado 17 abril 2020]. Disponible en: http://www.gcamerica.com/products/operatory/ EQUIA_Forte/

36. VOCO GmbH. Materiales a base de ionómero de vidrio de Voco. Iono fil Plus. [Consultado 15 julio 2020] Disponible en: http://onipo.com.mx/wp-content/ uploads/2018/02/folleto-23.pdf

37. 3M ESPE. Ketac Molar Easy Mix. [citado el 06 octubre 2019]. Disponible en: https://multimedia.3m.com/ mws/media/281923O/ketac-molar-easymix-technical-product-profile.PDF

38. Europe GC Dental. Fuji IX GP Extra Capsule. [Consultado 12 julio 2020] Disponible en: https://europe.gc. dental/sites/europe.gc.dental/files/products/downloads/ fujiggp/ifu/IFU_Fuji_IX_GP_EXTRA_Capsules_W. pdf

39. Grossi J, Nunes R, Dias A, Coelho S. Glass hybrid restorations as an alternative for restoring hypomineralized molars in the ART model. BMC Oral Health. 2018;18:65. DOI: 10.1186/s12903-018-0528-0

40. Mount GC. An Atlas of Glass Ionomer Cements: Clinician's Guide. 3ra edición, Londres: Edit. Martin Dunitz; 2002. 